\title{
Expect the Unexpected: Materials Research Transforming the Future
}

As someone who has made a 50-plusyear career of materials research, it is a real pleasure to have the opportunity to tell the members of the materials community about some of the exciting materials research programs and activities of the National Science Foundation (NSF). NSF supports research that has the potential to transform not just fields of science and engineering, but ultimately society itself. NSF is very different from the other U.S. federal agencies that fund research, because we are not restricted to a particular field or mission-our mandate is simply to support any and all fields of science and engineering. Our role is to foster innovation in its

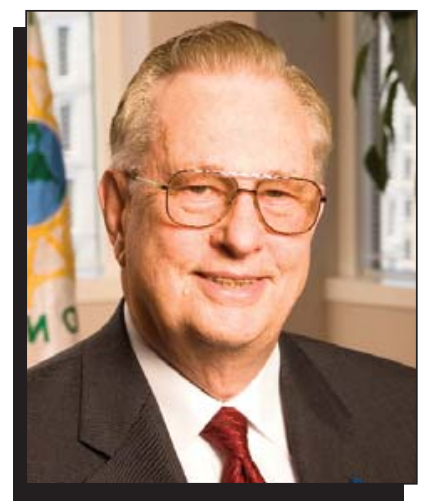

Arden L. Bement, Jr. infancy, to look for science that develops new concepts and establishes new interdisciplines. Experience has taught us that our investment is best spent when we challenge old paradigms and ask ourselves what we could be doing differently.

NSF's investments in basic research translate to a secure economic future for the people of the United States, and for the stability of the global economy as a whole. We know that the discoveries of today become the innovations that feed into the high-tech economy of tomorrow, just as the students of today become the highly skilled workforce that drives that economy. Government investments in 
high-risk, high-reward basic research are essential to the companies that develop the technologies that enrich the lives of people around the world.

Last month's special issue of the MRS Bulletin (April 2008) addressed one of the most critical problems of the 21st century: energy. Energy is inextricably coupled to climate change and the choices human beings can make to meet the challenges that lie ahead. Our society is currently at a crossroads in policies for energy production and usage. Materials science is relevant to every aspect of energy: from developing new technologies for harvesting solar, geothermal, wind, ocean, and nuclear energy, to finding new ways of storing energy, to creating devices that consume energy wisely. Nowhere is transformative thinking more critical than in the issue of energy and sustainability. How will we generate the energy of the future? Will it be generated locally with minimal transmission losses? Can we find clean ways of powering our transportation systems that do not generate greenhouse gases or other substances detrimental to the environment? Can we reduce our energy and resource consumption, and, in turn, our footprint on the world's ecosystems?

A recent example of NSF-funded research highlights an approach to energy generation that is transformative in its very simplicity (see Figure 1). Zhong Lin Wang, a professor at the Georgia Institute of Technology, has built a nanoscale power generator that harvests mechanical energy from its environment, transforming the kinetic energy into electricity. Zinc oxide nanowires produce a tiny amount of current when they bend, due to the coupling of their piezoelectric and semiconducting properties. Wang's group constructs power generators by growing arrays of nanowires on top of a solid layer of zinc oxide, which is used to make electrical contact with the nanowires. A zigzag-textured platinum-coated silicon counter electrode placed in close proximity to the nanowires collects the charge created when the nanowires flex and contact the points of the electrode. The nanogenerators passively collect energy from their environment in the form of acoustic vibrations and even biological hydraulic energy (such as from the motion of blood in a living organism). This approach could one day make possible self-powered implantable biomedical devices, wireless sensors, and other miniaturized electronic applications.

Sometimes, transformative research involves breathing new life into an existing technology. Researchers at the Massachusetts Institute of Technology's Mate- rials Research Science and Engineering Center, in collaboration with researchers at the State University of New York at Stony Brook, have modified the structure of the crystal commonly used in lithiumion batteries to enable faster recharging times (see Figure 2). Through a combination of fundamental computer modeling and sophisticated materials characterization, the research team, led by Michael Rubner, sought a directed approach to creating a crystal that would retain the high charge capacity of the previous material while improving upon its ability to charge quickly. This improvement is particularly attractive in applications such as electric vehicles. The re-engineered crystal exhibits more than a tenfold increase in its storage capacity under high rates of charge and discharge.

Transformative applications of technology can result from the materials properties of the technology itself. Through the Small Business Innovation Research (SBIR) program, NSF provided early stage funding to a small company that is now emerging as a promising manufacturer of flexible polymer solar cells. Lowell, Mass.-based Konarka Technologies, Inc. uses light-absorbing semiconducting polymers as the active material in their photovoltaic devices. The inherent flexibility of the polymers opens a wide range of possible applications for these
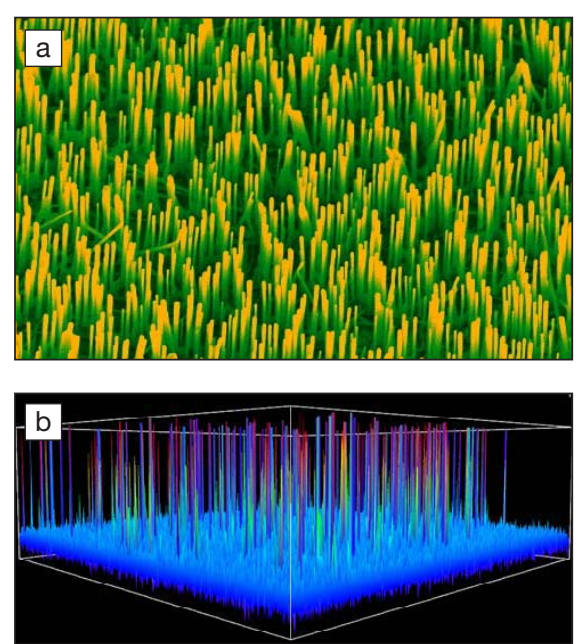

Figure 1. (a) Scanning electron microscope image (false color) of an array of $\mathrm{ZnO}$ nanowires. (b) Output voltage of an array of nanowires (vertical scale); the voltage was recorded by a platinumcoated atomic force microscope tip scanned across the nanowire array in contact mode. (Credit: Z.L. Wang, Georgia Tech. NSF award number CAREER DMR-Metals-9733160.) solar cells that would be impossible with the rigid silicon solar cells we are all familiar with, including integration into fabric for clothing or handbags capable of powering hand-held electronic devices. Konarka's flexible plastic solar cells are just one example of an energy technology that has emerged from basic research on the properties of semiconducting polymers. At NSF, we are looking to fund the basic research that leads to the next idea that transforms the energy marketplace.

Transformative thinking should not stop in the laboratory-at NSF, we are interested in finding new ways to transform science and engineering education. How could we change our current education system to capture all students who might excel at careers in math and science? How can we create new pathways for students to enter the pipeline of science and engineering education through outreach to two-year colleges, women's colleges, historically black colleges and universities, tribal colleges, and other lesstraditional institutions? We need to find ways to include people from underrepresented groups who have been largely excluded from participating in science and engineering careers, including women and persons with physical disabilities. We can make careers in materials science and engineering more enticing for all students by emphasizing how the innovations resulting from materials research lead to a better quality of life and improved economic development for people all over the world.

We need to be more creative in the way we reach out to young people. Materials science and engineering are especially attractive because they result in tangible products and processes that have changed the way in which people live and think. Outreach activities not only keep the public informed of the benefits of science and technology to society, they also give us the opportunity to stimulate the interest of young people, by showing them how they too can have a positive impact in the world through a career in science or engineering.

Rensselaer Polytechnic Institute's Molecularium ${ }^{\mathrm{TM}}$ is an example of NSFfunded outreach designed to transform the way young people think about the world on a molecular scale (see Figure 3). The Molecularium is a digital-dometheater that takes the audience on a ride through the world of atoms and molecules. A cast of atomic characters reveals the wonders of the universe on the nanoscale, capturing young imaginations while illustrating basic scientific concepts.

The increasing complexity of materials 


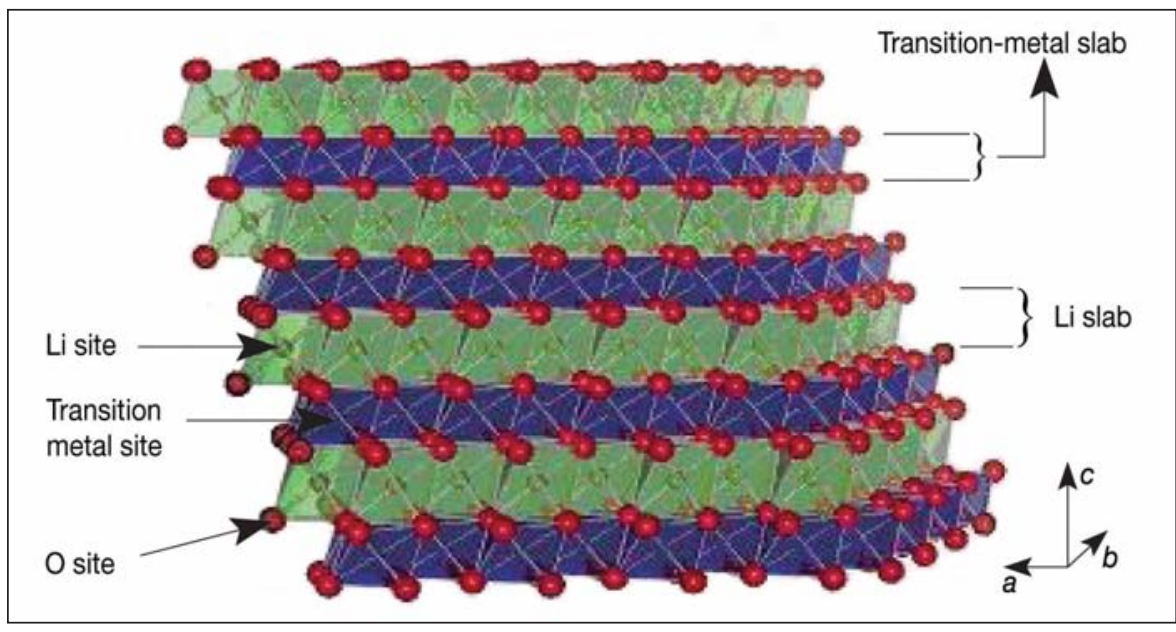

Figure 2. Structure of modified electrode material comprised of layers of nickel and manganese (blue layers) separated from lithium layers (green) by oxygen atoms (red). In charging and discharging of a lithium-ion battery, Li ions migrate in and out of the Li slab. (Credit: Gerd Ceder, MIT. NSF award number DMR-MRSEC-0213282.)

research necessitates collaboration among researchers in diverse fields, yielding new interdisciplines, and the need for sophisticated instrumentation and research centers. NSF's Materials Research Science and Engineering Centers (MRSEC) program has active participants at 26 universities across the country. MRSECs allow researchers to tackle large research projects that, like materials research itself, are highly inter- and multidisciplinary. MRSECs also provide important educational and research opportunities for undergraduate and graduate students, and high school students and teachers, through programs such as Research Experience for Undergraduates and Research Experience for Teachers. Another program, unique to the Division of Materials Research (DMR), is the Partnerships for Research and Education for Materials (PREM) program which pairs DMR funded researchers and educators with students and faculty at minority-serving institutions. The Nanoscale Science and Engineering Centers (NSECs) program is jump-starting discoveries and student training in nanoscience at universities across the United States, and is another important Centers program fueling materials research at universities across the country.

At NSF, we are particularly interested in forging collaborations and cooperation among scientists and engineers around the world, and preparing students to be agile thinkers in the global environment. The Division of Materials Research has established The Materials World Network (MWN), in which U.S. researchers

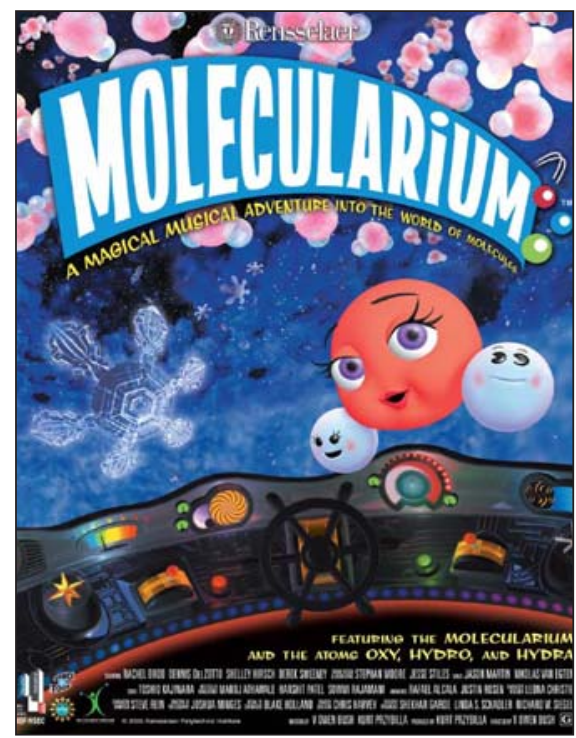

Figure 3. The Molecularium project is the flagship effort of the educational and outreach program of Rensselaer's NSF-funded Nanoscale Science and Engineering Center for Directed Assembly of Nanostructures. (NSF award number DMR-NSEC-0117792.) and educators partner with international collaborators on project proposals, which are funded in partnership with our international sister research funding agencies. Since 2001, over 180 awards have been given for a total of some $\$ 67$ million in funding. The International Materials Institutes program through DMR provides universities with the chance to become world leaders in materials research and to provide students with the opportunity to forge international ties early in their careers.

The NSF Web site can be accessed at www.nsf.gov for more information about the many funding opportunities available to materials researchers and educators. The Division of Materials Research is an obvious place to start, but chemistry, engineering, biology, and physics are just a handful of program areas that harbor potential overlap with the interests of materials researchers. The SBIR program can help researchers take innovative materials ideas to the marketplace.

We know that computational materials and cyber-enabled discovery will become increasingly essential to materials research, underpinning new discoveries and innovations, including new "materials by design" through petascale computing, metamaterials, and materials far from equilibrium. Materials research will continue to test the limits of what we can "see"whether it is testing the ultimate limits of measurement, devising new phenomena for imaging the unseen, or using computer simulations to enable us to visualize what cannot be seen directly. "Green" materials of the future may help us achieve "cradleto-cradle" use of resources, enabling us to give the Earth a reprieve from our current resource-hungry manufacturing processes. As we at NSF look to the horizon, we see an ever-expanding portfolio of funding opportunities for materials researchers. The President recently requested a 13\% increase in our fiscal year 2009 budget, and we are hopeful that Congress will also see the importance of increasing its support of our mission. We are excited by what the future holds for materials science and engineering. Our mantra for materials research is, "expect the unexpected." We cannot be sure of what tomorrow's great discoveries will be, but we know they will be amazing. ARDEN L. BEMENT, JR.

Arden L. Bement, Jr., is director of the National Science Foundation. 